\title{
Successful Management of an Extremely Premature Infant with Congenital Candidiasis
}

\author{
Sota Iwatani, MD ${ }^{1, *}$ Yuko Murakami, MD ${ }^{1,2, *}$ Masami Mizobuchi, MD, PhD ${ }^{1}$ \\ Kazumichi Fujioka, MD, PhD ${ }^{1}$ Keiko Wada, MD, PhD ${ }^{1}$ Hitomi Sakai, MD ${ }^{1}$ Seiji Yoshimoto, MD, PhD ${ }^{1}$ \\ Hideto Nakao, MD, $\mathrm{PhD}^{1}$
}

${ }^{1}$ Department of Neonatology, Hyogo Prefectural Kobe Children's Hospital Perinatal Center, Suma-Ku, Kobe-Shi, Hyogo, Japan

2 Department of Obstetrics and Gynecology, Okayama University Graduate School of Medicine, Kita-Ku, Okayama-Shi, Okayama, Japan

*These authors contributed equally to this work.

Am J Perinatol Rep 2014;4:5-8.

\begin{abstract}
Address for correspondence Sota Iwatani, MD, Department of Neonatology, Hyogo Prefectural Kobe Children's Hospital Perinatal Center, 1-1-1 Takakuradai, Suma-Ku, Kobe, Hyogo 654-0081, Japan (e-mail: iwatani_kch@hp.pref.hyogo.jp).
\end{abstract}

\begin{abstract}
Keywords

- congenital candidiasis

- candida albicans

- chorioamnionitis

- erythematous papule

- extremely premature infant

Congenital candidiasis, which presents with a variety of clinical symptoms, is very rare in both term and preterm infants, and less than 100 neonatal cases have been reported in the medical literature. We describe the case of an extremely premature infant with congenital candidiasis, who was successfully treated and survived without major sequelae. A male infant was born at 25 weeks' gestation (weight, $834 \mathrm{~g}$ ). He exhibited diffuse erythematous papules. Samples of his skin, pharyngeal mucus, gastric fluid, and tracheal aspirate were found to be Candida albicans-positive while blood cultures were negative. Further histopathological examinations revealed that Candida albicans mycelia had invaded the umbilical cord. After prompt antifungal therapy, the patient's skin lesions improved markedly, and he was discharged from hospital without any major complications. This report highlights the importance of characteristic skin lesions for the early diagnosis of Candida infections, especially in extremely premature infants.
\end{abstract}

Although cervicovaginal Candida infections occur in 20 to $25 \%$ of pregnancies, ascending infections only account for $0.8 \%$ of such infections, and they rarely cause chorioamnionitis. ${ }^{1}$ Congenital candidiasis is very rare in both term and preterm infants, with less than 100 neonatal cases having been published in the medical literature. ${ }^{2}$ In addition, congenital Candida infections are associated with intrauterine death or death in the immediate newborn period from disseminated disease, and the more premature a newborn infant is the greater the risk of death. ${ }^{3}$ We report the case of an extremely premature infant with congenital candidiasis, who was successfully treated and survived without major sequelae.

\section{Case Report}

A 28-year-old, gravida 1, para 1, mother was referred to our medical center at 22 weeks' gestation because of signs of

received

July 5,2013

accepted after revision

September 16, 2013

published online

December 12, 2013 preterm labor. The pregnancy had been uncomplicated, and the mother had no history of Candida vaginitis; tobacco, alcohol, or intrauterine conceptive device use; human immunodeficiency virus infection; or cervical cerclage.

Her vital signs were normal, and a blood examination revealed a white blood cell count of $12.5 \times 10^{9} / \mathrm{L}$ and a Creactive protein level of $0.43 \mathrm{mg} / \mathrm{dL}$. The discharge from her uterine cervix was cultured. Magnesium sulfate tocolysis was initiated to prevent premature labor, and ampicillin therapy was also administered. In addition, $12 \mathrm{mg}$ of betamethasone were intravenously administered for 2 days to enhance fetal lung maturity. However, at 25 weeks and 3 days' gestation active labor commenced suddenly, resulting in spontaneous vaginal delivery "en caul."

A male infant weighing $834 \mathrm{~g}$ was delivered with Apgar scores of 2 and 5 at 1 and 5 minutes, respectively. A physical examination demonstrated a premature infant
Copyright $\odot 2014$ by Thieme Medical Publishers, Inc., 333 Seventh Avenue, New York, NY 10001, USA. Tel: +1(212) 584-4662.
License terms

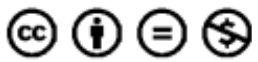

10.1055/s-0033-1358766. ISSN 2157-6998. 


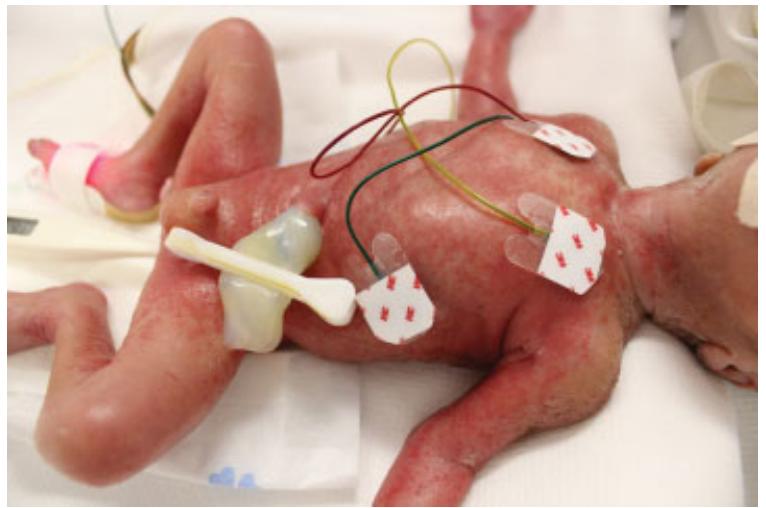

Fig. 1 At birth, diffusely distributed erythematous papules and pustules were present on the infant's face, trunk, and extremities.

in respiratory distress. The infant was intubated, and an endotracheal surfactant was administered. He had diffusely distributed erythematous papules and pustules on his face, trunk, and extremities ( - Fig. 1). His total blood cell count included a total leukocyte count of $4.3 \times 10^{9} / \mathrm{L}$ with $7.5 \%$ band forms, $43.0 \%$ polymorphonuclear leukocytes, and $33.5 \%$ lymphocytes, and a platelet count of $255 \times 10^{9} / \mathrm{L}$. A laboratory examination revealed a C-reactive protein level of $0.26 \mathrm{mg} / \mathrm{dL}$ and an immunoglobulin M (IgM) level of 3 $\mathrm{mg} / \mathrm{dL}$. A blood culture was negative. Culture samples obtained from the skin, pharyngeal mucus, gastric fluid, and tracheal aspirates were subsequently found to be positive for Candida albicans. Empiric antibiotic therapy with flomoxef was initiated according to our routine protocol. As the infant's skin lesions were characteristic of Candida infection, intravenous fluconazole ( $4 \mathrm{mg} / \mathrm{kg}$ ) was promptly administered. Clotrimazole was also used to treat the patient's skin lesions. After 1 day, his erythematous papules and pustules had markedly improved. Fluconazole was administered every third day for the first 7 days of life, which resulted in improvements in the patient's skin lesions and laboratory data. No major complications, such as patent ductus arteriosus, intraventricular hemorrhage, or fungal infection recurrence, were observed during the neonatal period. The infant was successfully extubated after 42 days of mechanical ventilation and placed on supplemental oxygen for 2 weeks, before being weaned onto room air.

The infant was discharged on the 90th day after birth. Follow-up evaluations were performed in an outpatient setting until 12 months of life, during which he did not display any abnormal symptoms or developmental delays.

A histopathological examination revealed: (1) acute chorioamnionitis combined with funisitis and (2) the invasion of Candida albicans mycelia into the umbilical cord (-Fig. 2). Culture samples obtained from the uterine cavity at delivery were subsequently found to be positive for Candida albicans. On the basis of these findings, we diagnosed threatened premature delivery combined with chorioamnionitis, funisitis, and neonatal congenital candidiasis caused by Candida albicans.

\section{Discussion}

Congenital candidiasis is very rare in both term and preterm infants, despite the high prevalence (20-25\%) of Candida vulvovaginitis during pregnancy. ${ }^{1-4}$ Congenital candidiasis causes a variety of clinical symptoms, ranging from diffuse erythematous skin eruptions with or without vesicles and pustules to systemic disease, in which the lungs are usually affected and the skin may or may not be affected. ${ }^{1-4}$ In addition, hematological abnormalities, for example, an increased number of immature granulocytes, have also been reported to occur in congenital candidiasis. ${ }^{5,6}$ In the present case, the infant exhibited characteristic skin lesions and transient neutropenia at birth, which aided the recognition and treatment of his Candida infection. However, he demonstrated normal C-reactive protein and IgM levels at birth. Therefore, the presence of skin lesions is considered to be important for the early detection for Candida infections, especially in very premature infants.

It is generally accepted that congenital candidiasis is acquired in utero via ascending infections although the exact mechanism by which this occurs is currently unknown. Four possible explanations have been proposed for Candida species reaching the upper genital tract and causing chorioamnionitis: (1) direct invasion from the vagina after premature rupture of membranes; (2) the resealing of a ruptured amnion; (3) migration across an intact amnion; and (4) the

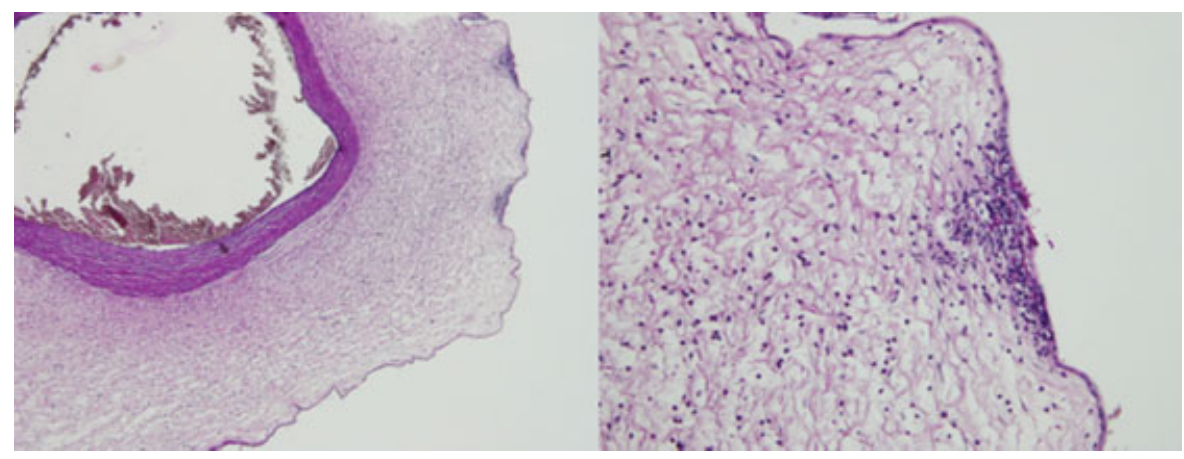

Fig. 2 Histological view of the umbilical cord obtained using periodic Acid-Schiff stain (left, $\times 40$; right, $\times 200$ ). Candida albicans mycelia had invaded into the umbilical cord. 
presence of foreign bodies, such as an intrauterine contraceptive device or cerclage, in the genital tract. ${ }^{4}$ It is worth noting that most reported cases of congenital candidiasis have involved clinically intact chorioamniotic membranes. ${ }^{1-3,7}$ In the present case, the infant was delivered en caul, and the mother had no history of foreign bodies, amniocentesis, or premature rupture of membranes. However, histopathological examinations of the placenta revealed that Candida albicans mycelia had invaded the umbilical cord, suggesting that Candida species had crossed the intact chorioamniotic membranes. Surprisingly, only a few reports have described the invasion of Candida albicans mycelia into the placenta or umbilical cord. ${ }^{8,9}$ Precise examinations of placental pathology might be critical for clarifying the pathogenesis of congenital candidiasis.

Preterm infants suffering from Candida infection are at great risk of serious morbidities and mortality. ${ }^{3,7}$ Prophylactic systemic antifungal treatment has been reported to prevent mortality and morbidities in very low birth weight infants. ${ }^{10}$ In our case, as antifungal therapy was promptly administered in response to the patient's characteristic skin lesions the infant survived and did not suffer any major complications during the neonatal period. Moreover, he did not display any abnormalities or developmental delays at 12 months of age. These findings suggest that early prophylactic antifungal treatment reduces the risk of morbidities and mortality.

In a nationwide survey conducted in Israel, it was found that candidal sepsis, in contrast to bacterial sepsis, was independently associated with the development of bronchopulmonary dysplasia (BPD). ${ }^{11}$ In addition, Pinhat suggested that fungal colonization itself is a risk factor for the BPD development. ${ }^{12}$ In our case of congenital candidiasis, the infant's tracheal aspirate cultures were found to be which positive for Candida albicans; however, he was successfully weaned onto room air at a postmenstrual age of 34 weeks. These findings suggest that early antifungal treatment might be useful for reducing the risk of BPD development.

In conclusion, we have described the case of an extremely premature infant with congenital candidiasis, who was successfully treated and survived without suffering any major sequelae. This report highlights the importance of characteristic skin lesions for the early diagnosis of Candida infections, especially in extremely premature infants. Despite the high mortality rate of Candida infections in preterm infants, early recognition and treatment could increase the chance of a favorable outcome.

\section{Acknowledgment}

We thank Dr. Makiko Yoshida of the Department of Pathology, Hyogo Prefectural Kobe Children's Hospital, for her invaluable assistance.

\section{Conflict of Interest}

The authors have no conflicts of interest to declare.

\section{References}

1 Van Winter JT, Ney JA, Ogburn PLJr, Johnson RV. Preterm labor and congenital candidiasis. A case report. J Reprod Med 1994;39(12): 987-990

2 Darmstadt GL, Dinulos JG, Miller Z. Congenital cutaneous candidiasis: clinical presentation, pathogenesis, and management guidelines. Pediatrics 2000;105(2):438-444

3 Johnson DE, Thompson TR, Ferrieri P. Congenital candidiasis. Am J Dis Child 1981;135(3):273-275

4 Rode ME, Morgan MA, Ruchelli E, Forouzan I. Candida chorioamnionitis after serial therapeutic amniocenteses: a possible association. J Perinatol 2000;20(5):335-337

5 Wolach B, Bogger-Goren S, Whyte R. Perinatal hematological profile of newborn infants with candida antenatal infections. Biol Neonate 1991;59(1):5-12

6 Ozkiraz S, Tarcan A, Gokmen Z, Gürakan B, Bilezikci B, Ozbek N. Invasive Candida albicans infection mimicking leukemia in a neonate. J Matern Fetal Neonatal Med 2007;20(7):555-557

7 Cotch MF, Hillier SL, Gibbs RS, Eschenbach DA; Vaginal Infections and Prematurity Study Group. Epidemiology and outcomes associated with moderate to heavy Candida colonization during pregnancy. Am J Obstet Gynecol 1998;178(2):374-380

8 Berry DL, Olson GL, Wen TS, Belfort MA, Moise KJ Jr. Candida chorioamnionitis: a report of two cases. J Matern Fetal Med 1997; 6(3):151-154

9 Ito F, Okubo T, Yasuo T, et al. Premature delivery due to intrauterine Candida infection that caused neonatal congenital cutaneous candidiasis: a case report. J Obstet Gynaecol Res 2013;39(1): 341-343

10 Manzoni P, Stolfi I, Pugni L, et al; Italian Task Force for the Study and Prevention of Neonatal Fungal Infections; Italian Society of Neonatology. A multicenter, randomized trial of prophylactic fluconazole in preterm neonates. N Engl J Med 2007;356(24): 2483-2495

11 Makhoul IR, Bental Y, Weisbrod M, Sujov P, Lusky A, Reichman B; Israel Neonatal Network. Candidal versus bacterial late-onset sepsis in very low birthweight infants in Israel: a national survey. J Hosp Infect 2007;65(3):237-243

12 Pinhat EC, Borba MG, Ferreira ML, et al. Fungal colonization in newborn babies of very low birth weight: a cohort study. J Pediatr (Rio J) 2012;88(3):211-216 